\title{
COMMUNICATIONS
}

\author{
DE LA FACULTÉ DES SCIENCES \\ DE L'UNIVERSITÉ D'ANKARA
}

Série A: Mathématiques, Physique et Astronomie

On $|\mathbf{C}, 1|$ Summability Factors of Fourier Series

by

RAJIV SINHA

15

Faculté des Sciences de l'Université d'Ankara Ânkara, Turquie 


\title{
Communications de la Faculté des Sciences de l'Université d'Ankara
}

\author{
Comité de Rédaction de la Série $A_{3}$ \\ C. Uluçay, E. Erdik, N. Doğan
}

Secrétaire de publication

N. Gündüz

La Revue "Communications de la Faculté des Sciences de l'Univereité d'Ankara" est un organe de publication englobant toutes les disciplines scientifiques représentées à la Faculté: Mathématiques pures et appliquées, Astronomie, Physique et Chimie théorique, expérimentale et techinique, Géologie, Botanique et Zoologie.

La Revue, á l'exception des tomes I, II, III, comprend de trois séries

Série A: Mathématiques, Physique et Astronomie.

Série B: Chimie.

Série C: Sciences naturelles.

En principe. la Revue est réservée aux mémoires originaux des membres de la Faculté. Elle accepte cependant, dans la mesure de la place disponible, les communications des auteurs étrangers. Les langues allemande, anglaise et française sont admises indifféremment. Les articles devront être accompagnés d'un bref sommaire en langue turque. 


\title{
On $|\mathrm{C}, 1|$ Summability Factors of Fourier Series
}

\author{
RAJIV SINHA
}

Department of Mathematics, Kurukshetra University, Kurukshetra (India)

Let $f(t)$ be a periodic function with period $2 \pi$ and integrable in the sense of Lebesgue over $(-\pi, \pi)$, then its Fourier series is

$$
\begin{gathered}
f(t) \sim \frac{1}{2} a_{0}+\sum_{n=1}^{\infty}\left(a_{n} \cos n t+b_{n} \sin n t\right) \equiv \\
\equiv A_{n}(t)
\end{gathered}
$$

Concerning the | C, 1 | summability of Fourier series Fu Cheng Hsiang (Pac. J. of Maths. Vol. 33, No. 1, 1970) has prove dsome therems.

Our aim is to prove the same theorems under a weaker condition. Our main theorem is as follows:

Theorem. If

$$
\int_{x}^{\pi} \frac{|\varphi(u)|}{u} \mathbf{u} u=0\left(\log \frac{1}{x}\right)
$$

as $\mathbf{t} \rightarrow+0$, then the series

$$
\Sigma \frac{\text { An (xo) }}{\mathbf{n}^{\alpha}}
$$

is $|\mathrm{C}, 1|$ summable, $\alpha>0$.

1. Let $f(t)$ be a periodic function with period $2 \pi$ and integrable in the sense of Lebesgue over $(-\pi, \pi)$, then its Fourier series is

(1.1) $f(t) \sim \frac{1}{2} a_{0}+\sum_{n=1}^{\infty}\left(a_{n} \cos n t+b_{n} \operatorname{sinn} t\right)=\sum_{n=0}^{\infty} A_{n}(t)$ 
and the conjugate series

$$
\Sigma\left(b_{n} \operatorname{cosn} t-a_{n} \sin n t\right) \equiv \sum_{n=1}^{\infty} B_{n}(t)
$$

Suppose $s_{n}$ denotes the partial sum of the infinite series $\Sigma a_{n}$ and let $S_{n}^{\alpha}$ and $t_{n}^{\alpha}$ denote the nth Cesaro mean of order $\alpha$, $(\alpha>-1)$ of the sequences $\left\{s_{n}\right\}$ and $\left\{n a_{n}\right\}$ respectively. The infinite series $\Sigma a_{n}$ is said to be summable $|C, \alpha|$ if the sequence $\left\{s_{\mathbf{n}}{ }^{\alpha}\right\} \approx B V$, i.e.,

$$
\sum_{n=1}^{\infty}\left|s_{n}^{\alpha}-s_{n-1}^{\alpha}\right|<\infty
$$

We know that

$$
\mathbf{t}_{n}^{\alpha}=\mathbf{n}\left(\mathrm{s}_{n}^{\alpha-s_{n_{-1}}^{\alpha}}\right)
$$

so

$$
\Sigma\left|T_{\mathbf{n}}^{\alpha}\right| / \mathbf{n}<\infty
$$

We shall use throughout this paper the following notations:

For a fixed point $x_{0}$, we write

$$
\begin{aligned}
& \varphi(t)=\varphi \mathbf{x}_{0}(t)=\mathbf{f}\left(\mathbf{x}_{0}+\mathbf{t}\right)+\mathbf{f}\left(\mathbf{x}_{0}-t\right)-2 \mathbf{f}\left(\mathbf{x}_{0}\right) \\
& \Phi(t)=\int_{0}^{t}|\varphi(u)| \mathbf{d u} ;
\end{aligned}
$$

and

$\Psi(t)=\int_{0}^{t}|\psi(u)| d u \equiv \int_{0}^{t}\left|f\left(x_{0}+u\right)-f\left(x_{0}-u\right)\right| d u$ and moreover

$\log ^{k} n=\log \left(\log ^{k-1} n\right)$ and $\log ^{2} n=\log (\log n)$.

2. Generalizing the previous result of Chow [1], Hsiang [2] quite recently proved the following theorems:

Theorem A. If

$$
\Phi(\mathrm{t})=\int_{0}^{\mathrm{t}}|\varphi(\mathrm{u})| \mathbf{d u}=\mathbf{0}(\mathrm{t})
$$

as $\mathrm{t} \longrightarrow+0$, then the series

$$
\Sigma A_{n}\left(\mathbf{x}_{0}\right) / \mathbf{n}^{\alpha}
$$

is summable | C, 1 | for every $\alpha>0$. 
Theorem B. If

$$
\Phi(\mathbf{t})=0\left(\frac{t}{\prod_{\mu=1}^{k} \log _{\mu} \frac{1}{t}}\right)
$$

as $\mathbf{t} \longrightarrow+\mathbf{0}$, then the series

$$
\sum_{n=n_{0}}^{\infty} \frac{A_{n}\left(x_{0}\right)}{\left(\prod_{\mu=1}^{k-1} \log ^{\mu} n\right)(\log n)^{1+\epsilon}},\left(\log ^{k} n_{0}>0\right)
$$

is summable $|\mathrm{C}, 1|$ for every $\varepsilon>0$.

Theorem C. If

$$
\Psi(\mathrm{t})=\int_{0}^{\mathrm{t}}|\psi(\mathrm{u})| \mathrm{du}=0(\mathrm{t})
$$

as $\mathbf{t} \longrightarrow+\mathbf{0}$, then the series

$$
\sum_{n=1}^{\infty} \frac{B_{n}\left(x_{0}\right)}{n^{\alpha}}
$$

is summable $|\mathrm{C}, 1|$ for every $\alpha>0$,

Theorem D. If

$$
\Psi(t)=\int_{0}^{t}|\psi(u)| d u=0\left(\frac{t}{\left(\prod_{\mu=1}^{k} \log ^{\mu} \frac{1}{t}\right)}\right)
$$

as $\mathrm{t} \longrightarrow+\mathrm{o}$, then the series

$$
\sum_{n=n_{0}}^{\infty} \frac{B_{n}\left(x_{0}\right)}{\left(\prod_{\mu=1}^{k-1} \log ^{\mu} n\right)\left(\log ^{k} n\right)^{1+\epsilon}},\left(\log ^{k} n_{0}>0\right)
$$

is summable $|\mathrm{C}, 1|$ for every $\varepsilon>0$.

It is easily seen [3] that, if (2.1) holds then

$$
\int_{t}^{\pi} \frac{|\varphi(u)|}{u} d u=0\left(\log \frac{1}{t}\right) \text { as } t \longrightarrow 0
$$


but it is not true conversely.

On the other hand, if (2.5) is true, then

$$
\int_{0}^{t}|\varphi(u)| d u=0\left(t \log \frac{1}{t}\right)
$$

and this result is best possible. Thus (2.6) is a weaker assertion than (2.1).

The object of this paper is to replace condition (2.1) by (2.5) which is a weaker one, in all the above mentioned theorems. Hence we prove the following:

Theorem 1. If

$$
\int_{t}^{\pi} \frac{|\varphi(u)|}{u} d u=0\left(\log \frac{1}{t}\right)
$$

as $\mathrm{t} \longrightarrow+\mathbf{0}$, then the series

$$
\Sigma \frac{A_{n}\left(x_{0}\right)}{n^{\alpha}} \text { is | C, } 1 \text { | summable. for every } \alpha>0 \text {. }
$$

Theorem 2. If

$$
\int_{t}^{\pi} \frac{|\varphi(u)|}{u} d u=0\left(\frac{\log \frac{1}{x}}{\prod_{\mu=1}^{k} \log ^{\mu} \frac{1}{t}}\right)
$$

as $\mathrm{t} \longrightarrow+\mathrm{o}$, then the series

$$
\sum_{n=n_{0}}^{\infty} \frac{A_{n}\left(x_{0}\right)}{\left(\sum_{\mu=1}^{\mathbf{I I}} \log ^{\mu} n\right)\left(\log ^{k} n\right)^{1+\epsilon}},\left(\log ^{k} n_{0}>0\right)
$$

is | C,1 | summable, for every $\varepsilon>0$.

Theorem 3. If

$$
\int_{t}^{\pi} \frac{|\dot{\psi}(u)|}{u} d u=0\left(\log \frac{1}{t}\right)
$$


as $\mathrm{t} \longrightarrow+0$, then the series

$$
\Sigma \frac{A_{n}\left(\mathbf{x}_{0}\right)}{n^{\alpha}}
$$

is $|\mathrm{C}, 1|$ summable for every $\alpha>0$.

Theorem 4. If

$$
\int_{t}^{\pi} \frac{|\psi(u)|}{u} d u=0\left(\frac{\left(\log \frac{1}{t}\right)}{\left(\sum_{\mu=1}^{k-1} \log ^{\mu} n\right)\left(\log ^{k}\right)^{1+\epsilon}}\right)
$$

as $\mathrm{t} \longrightarrow+0$, then the series

$$
\sum_{n=\mathbf{n}_{0}}^{\infty} \frac{B_{n}\left(x_{0}\right)}{\left(\prod_{\mu=1}^{\operatorname{lI}} \log ^{\mu} n\right)\left(\operatorname{lon}^{k} n\right)^{1+\epsilon}},\left(\log ^{k} n_{0}>0\right)
$$

is summable $\mid \mathrm{C}, 1$ for every $\varepsilon>0$.

3. For the proofs of these theorems, we require the following lemmas:

Lemma 1 [2]. Let

$$
S v(t)=\sum_{\nu=0}^{n} v \cos v t
$$

then

$$
\text { Su }(t)= \begin{cases}0\left(n^{2}\right) & (\text { for all } t) \\ 0(n / t) & (\text { nt } \geq 1)\end{cases}
$$

Lemma 2. [2].

$$
\mid \frac{1}{n+1}\left\{\sum_{v=1}^{n} \text { Sv (t) } \triangle \frac{1}{(u+2)^{\alpha}}\right\} \mid \leqq\left\{\begin{array}{c}
\frac{A^{*}}{\operatorname{tn}^{\alpha}}+\frac{\mathbf{A}}{n t^{1-\alpha}}(t>+>1) \\
\operatorname{An}^{1-\alpha} \quad(\text { for all t) }
\end{array}\right.
$$

${ }^{*} \mathrm{~A}$ is a finite constant but is nat necevanily the same at each oceuvvence. 


\section{Proof of Theorem 1}

We have

$$
A_{n}\left(x_{0}\right)=\frac{2}{\pi} \int_{0}^{\alpha} \varphi(t) \cos n t d t .
$$

Let $T_{n}\left(x_{0}\right)$ be the $n$th Cesaro mean of the first order of the sequence $\left\{\mathbf{n A}_{\mathbf{n}}\left(\mathrm{x}_{0}\right) / \mathbf{n}^{\alpha}\right\}$, then

$\frac{\pi}{2} T_{n}\left(x_{0}\right)=\int_{0}^{\pi} \varphi(t) \frac{1}{n+1} \sum_{v=0}^{n} \frac{(v+2) \cos (v+2) t}{(v+2)^{\alpha}} d t$.

Abel's transformation gives

$$
\begin{aligned}
& \frac{\pi}{2} T_{n}\left(x_{0}\right)=\int_{0}^{\pi} \varphi(t) \frac{1}{n+1}\left\{\sum_{\nu=0}^{n} S u(t) \triangle \frac{1}{(u+2)^{\alpha}}\right\} d t \\
& +\int_{0}^{\pi} \varphi(t) \frac{1}{n+1} \frac{S_{n}(t)}{(n+3)^{\alpha}} d t . \\
& \text { say. Thus, on writing } \quad=I_{1 n}+I_{2 n},
\end{aligned}
$$

$$
I_{1 n}=\int_{0}^{1 / n}+\int_{1 / n}^{\pi}=I_{3 n}+I_{4 n},
$$

say, we see that

$$
I_{3 n}=0\left(n^{1-\alpha} \int_{0}^{1 / n}|\varphi(t)| d t\right)=0(n-\alpha \cdot \log n)
$$

by conditions (2.6) and (2.7).

$$
\begin{aligned}
I_{4 n} & =0\left\{\frac{1}{n^{\alpha}} \int_{1 / n}^{\pi} \frac{|\varphi(t)|}{t} d t\right\}+0\left\{\frac{1}{n} \int_{n}^{\pi} \frac{|\varphi(t)|}{2-\alpha} d t\right\} \\
& =0\left\{\frac{1}{n^{\alpha}} \cdot(\log n)\right\}+0\left\{\frac{1}{n} \cdot n^{1-\alpha} \cdot(\log n)\right\}
\end{aligned}
$$

since $\int_{1 / n}^{\pi} \frac{|\varphi(t)|}{2-\alpha} d t \leq n^{1-\alpha} \int_{1 / n}^{\pi} \frac{|\varphi(t)|}{t} d t=0\left(n^{1-\alpha} \cdot \log n\right)$

Hence

$$
I_{4 n}=0\left\{n^{-\alpha}(\log n)\right\}
$$


by condition (2.7) of the theorem.

Now, as before, we write

$$
I_{2 n}=\int_{0}^{1 / n}+\int_{1 / n}^{\pi}=I_{5 n}+I_{6 n},
$$

say. Then,

$$
I_{s \mathbf{n}}=\left(\mathbf{n}^{1-\alpha} \int_{0}^{1 / n}|\varphi(t)| d t\right)=O\left(n^{-\alpha} \cdot \log n\right)
$$

and

$$
I_{6 n}=0\left\{n^{-\alpha} \int_{1 / n}^{\pi} \frac{|\varphi(t)|}{t} d t\right\}=0\left(n^{-\alpha} \log n\right),
$$

by the similar arguments as in the estimation of $I_{3 n}$ and $I_{4 n}$. But we have to show the convergence of $\Sigma T_{n}\left(x_{0}\right) / n$. And from the above analysis, it concludes that

$$
\begin{aligned}
\sum_{n=1}^{\infty} \frac{\left|T_{n}\left(x_{0}\right)\right|}{n} & \leq \frac{2}{\pi} \sum_{n=1}^{\infty} \frac{1}{n}\left\{\left|I_{3 n}\right|+\left|I_{3 n}\right|+\left|I_{5 n}\right|+\left|I_{6 n}\right|\right\} \\
& =0\left\{\sum_{n=1}^{\infty} \frac{\log n}{n^{1+\alpha}}\right\}=0(1) .
\end{aligned}
$$

This proves theorem 1 .

Proof of Theorem 2.

Let $T_{n}\left(x_{0}\right)$ be the nth Cesaro mean of first order of the sequence

$$
\left\{\mathbf{n} A_{n}\left(x_{0}\right) /\left({ }_{\mu=1}^{k-1} \log ^{\mu} n\right)\left(\log ^{k} n\right)^{1+\epsilon}\right\},(\varepsilon>0),
$$

where $k$ is a positive integer. Abel's transformation gives

$$
\begin{aligned}
& \frac{\pi}{2} T_{n}\left(x_{0}\right)=\int_{0}^{\pi} \varphi(t) \frac{1}{n+1}\left\{\sum_{\nu=1}^{n} S_{v}(t)\right.
\end{aligned}
$$

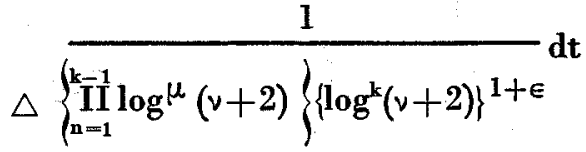




$$
\begin{aligned}
& +\int_{0}^{\pi} \varphi(t) \frac{1}{n+1}\left\{\sum_{\mu=1}^{k-1} \frac{S_{n}(t)}{\left.\log ^{\mu}(n+3)\right\}\left\{\log ^{k}(n+3)\right\}^{1+\epsilon}}\right. \\
& =I_{1 n}+I_{2 n},
\end{aligned}
$$

say, As before, we write

$$
I_{1 n}=\int_{0}^{1 / n}+\int_{1 / n}^{\pi}=I_{3 n}+I_{4 n}
$$

say, and

$$
I_{2 n}=\int_{0}^{1 / n}+\int_{1 / n}^{\pi}=I_{5 n}+I_{6 n}
$$

say. Since, for $v \geq \mathbf{n}_{0}$,

$$
\left|\Delta \frac{1}{\left(\sum_{\mu=1}^{\mathrm{i} I} \log ^{\mathrm{u}} v\right)\left(\log ^{\mu} v\right)^{1+\epsilon}}\right| \leq \frac{\mathbf{A}}{\left(\sum_{\mu=1}^{\mathrm{II}} \log ^{\mu} v\right)\left(\log ^{\mathrm{k}} v\right)^{1+\epsilon}}
$$

we obtain

$$
\begin{aligned}
& \left|\frac{1}{n+1} \sum_{v=0}^{n} S v(t) \triangle \frac{1}{\left(\prod_{\mu=1}^{k-1} \log ^{\mu}(v+2)\left(\log ^{k}(v+2)\right)^{1+\epsilon}\right.}\right| \leq \\
& \int \frac{A}{\mathbf{t}\left(\prod_{\mu=0}^{k-1} \log ^{\mu_{n}}\right)\left(\log ^{k} n\right)^{1+\epsilon}}+ \\
& \leq\left\{+\frac{A}{t^{2}\left(\sum_{\mu=1}^{k-1} \log ^{\mu} \frac{1}{t}\right)\left(\log ^{k} \frac{1}{t}\right)^{1+\epsilon}} \quad(n t \geq 1)\right. \\
& \frac{\text { An }}{\left(\prod_{\mu=1}^{k-1} \log ^{\mu} n\right)\left(\log ^{k} n\right)^{1+\epsilon}} \quad \text { (for all } t \text { ) }
\end{aligned}
$$


Now, by the conditions (2.6) and (2.8), we have

$$
\begin{aligned}
& I_{3 n}=0\left\{\frac{n}{\left(\sum_{\mu=1}^{k-1} \log ^{\mu} n\right)\left(\log ^{k} n\right)^{1+\epsilon}} \int_{0}^{1 / n}|\varphi(t)| d t .\right\} \\
& =0\left\{\frac{\log n}{\left(\sum_{\mu=1}^{k-1} \log ^{\mu} n\right)\left(\log ^{k} n\right)^{1+\epsilon}}\right\} \text {. } \\
& I_{4 n}=0\left\{\frac{1}{\left(\sum_{\mu=1}^{k} \bar{I}^{1} \log ^{\mu} n\right)\left(\log ^{k} n\right)^{1+\epsilon}} \int_{1 / n}^{\pi} \frac{|\varphi(t)|}{t} d t\right\}
\end{aligned}
$$

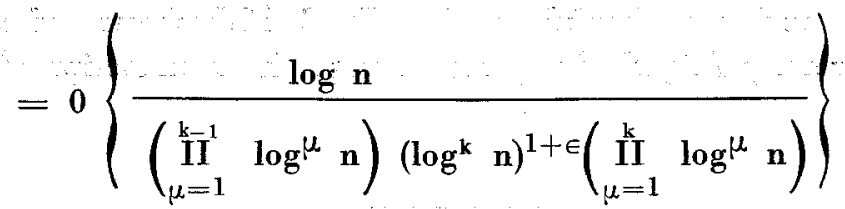

Finally

$$
\begin{aligned}
& I_{5 n}=0\left\{\frac{n}{\left(\sum_{\mu=1}^{I_{-1}} \log ^{\mu} n\right)\left(\log ^{k} n\right)^{1+\epsilon}} \int_{0}^{1 / n}|\varphi(t)| d t\right\} \\
& =0\left\{\frac{\log n}{\left(\prod_{\mu=1}^{\mathrm{II}} \log ^{\mu} \mathrm{n}\right)\left(\log ^{\mathrm{k}} \mathrm{n}\right)^{1+\epsilon}}\right\} \\
& I_{6 n}=0\left\{\frac{1}{\left(\prod_{\mu=1}^{k-1} \log ^{\mu} n\right) \operatorname{lo}\left(g^{k} n\right)^{1+\epsilon}} \int_{1 / n}^{\pi} \frac{|\varphi(t)|}{t} d t\right\}
\end{aligned}
$$




$$
=0\left\{\frac{\log \mathbf{n}}{\left(\int_{\mu=1}^{k-1} \log ^{\mu} \mathbf{n}\right)\left(\log ^{\mathrm{k}} \mathbf{n}\right)^{2+\epsilon}\left(\text { II }_{\mu=1}^{\mathrm{k}} \log ^{\mathrm{u}} \mathbf{n}\right)}\right\}
$$

Thus

$$
\begin{aligned}
& \sum_{n=1}^{\infty} \frac{\left|T_{n}\left(x_{0}\right)\right|}{n}=0\left\{\sum_{n=n_{0}}^{\infty} \frac{\log n}{\left(\prod_{\mu=1}^{k-1} \log ^{\mu} n\right)\left(\log ^{k} n\right)^{2+\epsilon}}\right\} \\
& =0 \text { (1) }
\end{aligned}
$$

Hence the proof of Theorem 2 is complete.

The proofs of Theorems 3 and 4 can be given on the same lines.

The authar iî thankful to Mr. D. C. Maheshwari of Muslim University Aligarh for going through the manuscript of the paper.

\section{REFERENCES}

[1] Chow,H.C., On the summability factors of Fourier series, J.London Math. Soc. 1941, 1941, 16 (1954), 215-220.

[2] Hsiang. F.C., On $|C, 1|$ summability facttors of a Fourier series at a given point, Pacific J. of Mathematics Vol. 33 No 1. 1970.

[3] Misra. C.L., On the determination of the jump of the function by its Fourire co-effi. cients. Vuart. J. Math. Oxford series 18 (1947), 129-134. 
Prix de l'abonnement annuel

Turquie : $15 \mathrm{TL}$; Etranger: $30 \mathrm{TL}$.

Prix de ce numéro : 5 TL (pour la vente en Turquie).

Prière de s'adresser pour l'abonnement à : Fen Fakültesi

Dekanlığı Ankara, Turquie.

Ankara Universitesi Basımevi. Ankara - 1976 